\title{
Evaluation of the Colin STBP-680 at rest and during exercise: an automated blood pressure monitor using R-wave gating
}

\author{
Vernon Bond Jr EdD, David R. Bassett Jr PhD, Edward T. Howley PhD, Jean Lewis PhD, \\ Ava J. Walker PhD, Pamela D. Swan PhD, Russell J. Tearney PhD* and \\ Richard G. Adams MD+ \\ Department of Human Performance and Sport Studies, University of Tennessee, Knoxville, Tennessee, USA, \\ ${ }^{*}$ Department of Physiology and Biophysics, Howard University, Washington, DC, USA and tDepartment of \\ Medicine, Howard University, Washington, DC, USA
}

\begin{abstract}
The application of automated blood pressure measurement during exercise has been limited by inaccuracies introduced by the effects of accompanying motion and noise. We evaluated a newly developed automated blood pressure monitor for measuring exercise blood pressure (Colin STBP-680; Colin, San Antonio, Texas, USA). The STBP-680 uses acoustic transduction with the assistance of the electrocardiogram $R$-wave to trigger the sampling period for blood pressure measurement. The automated monitor readings were compared with simultaneous technician mercury sphygmomanometric readings in the same arm. Blood pressure was measured in 18 men at rest and during exercise at $40 \% \dot{V}_{\mathrm{O}_{2} \text { peak, }}$ (low intensity), $70 \%$ $\dot{V}_{\mathrm{O}_{2} \text { peak }}$ (moderate intensity) and $\mathrm{Vo}_{2 \text { peak }}$ (high intensity) on the cycle ergometer. Mean(s.d.) systolic blood pressure difference between the automated monitor and mercury manometer readings at rest and during exercise at low, moderate and high work intensities were $3(0) \mathrm{mmHg}$, $3(2) \mathrm{mmHg}, 1(1) \mathrm{mmHg}$, and $0(11) \mathrm{mmHg}$ respectively (analysis of variance; $P>0.05$ ). Resting diastolic blood pressure obtained with the STBP-680 was similar to the mercury manometer readings $(78(10)$ versus $81(7) \mathrm{mmHg}$ $(P>0.05)$. Exercise diastolic pressure at the low level of work intensity was almost identical between the automated monitor and mercury manometer readings $(64(8)$ versus 65(10) $\mathrm{mmHg}$ (not significant)). Diastolic blood pressure readings between the STBP-680 and mercury manometer showed a greater difference at the moderate and high workloads $(11 \mathrm{mmHg}$ and $9 \mathrm{mmHg}$, respectively), but this difference was not significant $(P>0.05)$. The correlation for repeated submaximal exercise blood pressure determined by the STBP-680 was significant $(40 \%$ $\dot{V O}_{2 \text { peak }}$ systolic/diastolic, $\left.r=0.70 / 0.61, P<0.05 / 0.05\right)$, $70 \%{ }^{2} \mathrm{O}_{2 \text { peak }}$ systolic/diastolic, $r=0.75 / 0.71, P<0.05 /$ 0.05). These data show that the Colin STBP-680 accurately assessed systolic and diastolic pressure during rest and exercise, with the exception of diastolic pressure at moderate and high work intensity. Our findings show the STBP-680 has good potential in measuring exercise blood pressure, and is recommended as an alternative method
\end{abstract}

Address for correspondence: Vernon Bond, Jr, Louisiana State University, Department of Kinesiology, Baton Rouge, Louisiana, 70803, USA

(C) 1993 Butterworth-Heinemann Ltd 0306-3674/93/020107-06 for measuring blood pressure at low levels of work intensity.

Keywords: Automated blood pressure monitor, blood pressure, exercise

Monitoring of blood pressure during exercise testing is important to ensure the safety of the test and may provide important prognostic information ${ }^{1,2}$. Measurement of blood pressure during exercise is determined routinely by the standard method of auscultation using sphygmomanometry. It is difficult to assess blood pressure measurement during exercise because of motion and noise artifacts. Therefore, a reliable automated method of non-invasive determination of blood pressure has potential advantages ${ }^{3}$. Automated sphygmomanometers have been developed to overcome some difficulties of blood pressure measurement during exercise, but the performance of earlier automated blood pressure measuring devices were reported less than satisfactory when compared with mercury sphygmomanometric readings ${ }^{4}$. Recently, an automated blood pressure monitor (Colin STBP-680) that uses the electrocardiogram (ECG) $R$-wave in the discrimination of the Korotkoff sounds has become available commercially, and claims to provide a reliable means of measuring blood pressure during exercise. While many stress test laboratories have adopted the Colin STBP-680, only one study has validated the use of the machine against the traditional mercury sphygmomanometric readings during exercise conditions. A previous comparative study with this monitor ${ }^{5}$ used standard regression analysis to show agreement between automated and mercury manometer readings. The STBP-680 was reported to monitor relative changes in both systolic and diastolic blood pressures, but quantitatively overestimated systolic and diastolic blood pressure significantly. Further observation of the regression analysis showed that the STBP-680 and mercury manometer measurements agreed more closely at the higher level of work intensity. It may be more applicable to use statistical analysis testing the difference between mean values 
when showing the accuracy of the STBP-680 in measuring exercise blood pressure. The present study compares mean systolic and diastolic blood pressure values obtained by the Colin STBP- 680 with mercury sphygmomanometric readings during rest and at varying exercise intensities.

\section{Methods}

Informed consent was obtained from 18 men who agreed to participate in the investigation. The research proposal was approved by the University Review Committee for the Participation of Human Subjects. A description of the subjects is presented in Table 1.

Each subject initially performed a progressive exercise test on a mechanical braked Monark ergometer (Monark, Varberg, Sweden), assessing the blood pressure during rest (sitting position) and at maximum oxygen uptake $\left(\dot{V}_{2}\right.$ peak $)$. The ergometer test of $\dot{V}_{\mathrm{O}_{2} \text { peak }}$ consisted of pedalling at 50 r.p.m. at $25-\mathrm{W}$ increments every 2 min to maximum tolerance. Oxygen uptake and blood pressure were recorded during the last minute of each workload. Oxygen consumption was measured by an Applied Electrochemistry $\mathrm{S} 3-\mathrm{AO}_{2}$ analyser and $\mathrm{CD}-3 \mathrm{ACO}_{2}$ analyser (Ametek, Pittsburgh, Philadelphia, USA). Blood pressure was determined using a Colin STBP-680 automated monitor and mercury sphygmomanometer (W. A. Baum, Copiague, New York, USA). Approximately 1 week after the test of $\dot{V}_{\mathrm{O}_{2 p e a k}}$ each subject performed two submaximal tests on the ergometer working at $40 \% \dot{V}_{\mathrm{O}_{2} \text { peak }}$ and $70 \% \dot{V}_{\mathrm{O}_{2} \text { peak }}$. Duration of the submaximal workloads was $6 \mathrm{~min}$ each, with measurement of systolic and diastolic blood pressure recorded during the last minute of each workload. To assess the reproducibility of the automated monitor, submaximal exercise blood pressure was repeated over two days. The work outputs of $40 \% \dot{V}_{\mathrm{O}_{2} \text { peak }} 70 \% \dot{V}_{\mathrm{O}_{2} \text { peak }}$ and $\dot{V}_{\mathrm{O}_{2} \text { peak }}$ were defined as low, moderate and high work intensities respectively.

The mercury sphygmomanometer was calibrated and checked before the study. The STBP-680 was calibrated against the mercury manometer and was within $\pm 1 \mathrm{mmHg}$. The STBP-680 uses the ECG signals to trigger the microcomputer processing non-synchronous noise that allows isolation of Korotkoff sounds by acoustical transduction. The $\mathrm{CM}_{5}$ lead was used to obtain the ECG signal. The monitor automatically inflates and deflates the blood

Table 1. Physical characteristics of subjects

\begin{tabular}{lr}
\hline Variable & Mean(s.d.) \\
\hline Age (years) & $22.8(3.8)$ \\
Height $(\mathrm{cm})$ & $177.3(6.8)$ \\
Weight $(\mathrm{kg})$ & $75.9(8.4)$ \\
Skinfolds thickness $(\mathrm{mm})(\mathrm{sum}$ of triceps and iliac) & $16.2(4.9)$ \\
Peak oxygen uptake $\left(\mathrm{ml} \mathrm{min}^{-1} \mathrm{~kg}^{-1}\right)$ & $42.4(6.5)$ \\
Peak heart rate (beats $\left.\mathrm{min}^{-1}\right)$ & $182.7(8.3)$ \\
\hline
\end{tabular}

pressure cuff, and measures of systolic and diastolic blood pressure are displayed digitally. The pressure cuff of the STBP-680 was wrapped around the upper left arm of the subject with the dual sensors secured over the brachial artery. Using a Y-tube connector, blood pressure determinations were made in the same arm by the automated monitor and a technician blind to the monitor readings using the mercury manometer. The Korotkoff sounds were amplified from the cuff dual sensors into the headphone of the observer using the mercury manometer. The onset of Korotkoff sounds (phase I) was used for systolic blood pressure, and disappearance of sounds (phase V) was used for the diastolic blood pressure. To reduce experimental variability, the Korotkoff sound signals were determined by one technician.

\section{Statistical analysis}

Systolic and diastolic blood pressures were recorded during rest and at varying intensities of exercise. Differences between automated and mercury recordings were determined using a $2 \times 4$ factor analysis of variance. The Tukey post hoc test was used for significant main effect follow-up. Standard methods were used for calculation of correlation coefficients. Significance was established at the $P<0.05$ level.

\section{Results}

Blood pressures recorded by an observer using the mercury manometer and automatically by the STBP680 during rest and exercise are shown in Table 2.

At rest the mean(s.d.) systolic and diastolic blood pressure difference between the mercury manometer and automated monitor was $-3.2(0) \mathrm{mmHg}$ and $3(3) \mathrm{mmHg}$, respectively $(P>0.05)$. For resting systolic readings, the coefficient of variation was 9.4 with the mercury manometer and 9.5 with the STBP-680. For diastolic readings, coefficients of variation were 11.4 for the mercury manometer and 13.4 for the STBP-680. At the low intensity exercise level the mean(s.d.) difference between the automated monitor and mercury manometer pressures were systolic/diastolic: $3.0(2) / 2(2) \mathrm{mmHg}, P>0.05 /$ 0.05 . Systolic blood pressure at moderate work intensity differed by $1(1) \mathrm{mmHg}$ between the STBP680 and mercury manometer $(P>0.05)$. Diastolic blood pressure at moderate work intensity recorded by the automated monitor was $11(1) \mathrm{mmHg}$ higher

Table 2. Blood pressure values for Colin STBP-680 and mercury manometer during rest and exercise conditions (mean(s.d) $\mathrm{mmHg}$ )

\begin{tabular}{llllll}
\hline Variable & \multicolumn{2}{c}{ STBP-680 } & & \multicolumn{2}{c}{ Mercury manometer } \\
\cline { 2 - 3 } \cline { 5 - 6 } & Systolic & Diastolic & & Systolic & Diastolic \\
& & & & & \\
\hline Rest & $133(15)$ & $78(10)$ & & $130(15)$ & $81(7)$ \\
$40 \% \dot{V O}_{2 \text { peak }}$ & $149(10)$ & $64(8)$ & & $146(12)$ & $65(10)$ \\
$70 \% \dot{V O}_{2}$ peak & $183(16)$ & $71(9)$ & & $182(15)$ & $60(10)$ \\
$\dot{V O}_{2 \text { peak }}$ & $207(20)$ & $81(10)$ & & $207(9)$ & $72(11)$ \\
\hline
\end{tabular}


than the mercury manometer readings $(P>0.05)$. At the high work intensity systolic blood pressure measured with the STBP-680 was $207(20) \mathrm{mmHg}$ and the reading for the mercury manometer was 207(9) $\mathrm{mmHg}(P>0.05)$. Mean(s.d.) difference in diastolic pressure at the high work intensity between the STBP-680 and mercury manometer was $9(1) \mathrm{mmHg}(P>0.05)$. The coefficients of correlation for systolic and diastolic pressures for the automated monitor over two days and submaximal workloads were significant $\left(40 \% \dot{V} \mathrm{O}_{2 \text { peak }}\right.$ systolic/diastolic, $r=$ $0.70 / 0.61, P<0.05 / 0.05,70 \% \quad \dot{V} \mathrm{O}_{2 \text { peak }}$ systolic/ diastolic, $r=0.75 / 0.71, P<0.05 / 0.05$ ). The coefficients of correlation for systolic and diastolic pressures measured with the mercury manometer were also significant $\left(40 \% \dot{V}_{\mathrm{O}_{2} \text { peak }}\right.$ systolic/diastolic, $r=$ $0.79 / 0.69, P<0.05 / 0.05,70 \% \quad \dot{V} \mathrm{O}_{2 \text { peak }}$ systolic/ diastolic, $r=0.89 / 0.85, P<0.05 / 0.05)$.

\section{Discussion}

In the past, problems with motion and noise artifact have prevented reliable quantification of arterial pressure during exercise using automated blood pressure monitors. The Colin STBP-680, an automated blood pressure monitor, was recently developed to measure blood pressure during exercise. This automated monitor uses acoustic transduction and ECG-assisted microprocessing of nonsynchronous noise. We compared the pressures obtained by the STBP-680 with simultaneously recorded mercury sphygmomanometric readings during rest and cycle ergometer exercise at low, moderate and high work intensities. During rest the STBP-680 was found to provide mean systolic and diastolic blood pressure values within $3 \mathrm{mmHg}$ of those determined with the mercury manometer. The automated monitor satisfied the accuracy criteria of the Association for the Advancement of Medical Instrumentation (AAMI) which stipulates that the test device should not differ by more than $5 \mathrm{mmHg}$ from the mercury measurement, with a standard deviation not greater than $8 \mathrm{mmHg}^{6}$. Systolic blood pressure values at low, moderate and high levels of work intensity measured with the automated monitor were accurate when compared with the mercury manometer readings. The STBP- 680 diastolic readings correspond to the fifth-phase Korotkoff sounds, and were accurate compared with mercury manometer readings at the low level of work intensity. The STBP-680 determined diastolic pressure at the moderate and high work intensities varied from the mercury manometer by an average of $11 \mathrm{mmHg}$ and $9 \mathrm{mmHg}$, respectively, but this difference was not found to be statistically significant. One earlier study ${ }^{5}$ used regression analysis to examine the Colin STBP-680 ability to estimate exercise blood pressure and reported that the monitor tracked relative changes in systolic and diastolic pressures during exercise.

In conclusion, the Colin STBP-680 automated blood pressure monitor measuring resting and exercise blood pressure satisfied the accuracy criteria of the AAMI, except for diastolic readings at the moderate and high work intensities. The reliability and ease of use of this automated system were such that it could be a valuable asset in stress test laboratories. However, during exercise exceeding low work intensity, blood pressure readings by the automated monitor should be checked with the mercury manometer.

\section{References}

1 Bruce RA, Derouen TA, Peterson D et al. Non-invasive predictions of sudden cardiac death in men with coronary heart disease. Predictive value of maximal stress testing. $\mathrm{Am} \mathrm{J}$ Cardiol 1977; 39: 833-40.

2 Irving JB, Bruce RA, Derouen TA. Variations in and significance of systolic pressure during maximal exercise (treadmill) testing. Relation to severity of coronary artery disease and cardiac mortality. Am J Cardiol 1977; 39: 841-8.

3 Hossack KF, Gross BW, Ritterman JB, Kusumi F, Bruce RA. Evaluation of automated blood pressure measurement during exercise testing. Am Heart J 1982; 104: 1032-8.

4 Labarthe DR, Hawkins CM, Remington RD. Evaluation of performance of selected devices for measuring blood pressure. Am J Cardiol 1972; 32: 546-52.

5 Lightfoot JT, Tankersley C, Rowe SA, Freed AN, Fortney SM. Automated blood pressure measurements during exercise. Med Sci Sports Exerc 1988; 21: 698-707.

6 Association for The Advancement of Medical Instrumentation. American National Standard for Electronic or Automated Sphygmomanometers. Washington DC: AAMI, 1987. 